\title{
A Voxel-Based Assessment of Noise Properties in Computed Tomography Imaging with the ASiR-V and ASiR Iterative Reconstruction Algorithms
}

\author{
Patrizio Barca ${ }^{1,+}$, Daniela Marfisi ${ }^{1,+} \oplus$, Chiara Marzi $^{2}$, Sabino Cozza ${ }^{3}$, Stefano Diciotti ${ }^{2}$, Antonio Claudio Traino ${ }^{1}$ \\ and Marco Giannelli ${ }^{1, * \mathbb{B}}$ \\ 1 Unit of Medical Physics, Pisa University Hospital “Azienda Ospedaliero-Universitaria Pisana”, \\ 56126 Pisa, Italy; barca.patrizio@gmail.com (P.B.); marfisi.daniela@gmail.com (D.M.); \\ c.traino@ao-pisa.toscana.it (A.C.T.) \\ 2 Department of Electrical, Electronic and Information Engineering "Guglielmo Marconi", University of \\ Bologna, 47522 Cesena, Italy; chiara.marzi3@unibo.it (C.M.); stefano.diciotti@unibo.it (S.D.) \\ 3 Department of Radiology, Azienda USL Toscana Nord Ovest, 56025 Pontedera, Italy; \\ sabino.cozza@uslnordovest.toscana.it \\ * Correspondence: m.giannelli@ao-pisa.toscana.it \\ $+\quad$ These authors contributed equally to this study.
}

check for updates

Citation: Barca, P.; Marfisi, D.; Marzi, C.; Cozza, S.; Diciotti, S.; Traino, A.C.; Giannelli, M. A Voxel-Based Assessment of Noise Properties in Computed Tomography Imaging with the ASiR-V and ASiR Iterative Reconstruction Algorithms. Appl. Sci. 2021, 11, 6561. https://doi.org/ 10.3390/app11146561

Academic Editors: Ivan Veronese and Salvatore Gallo

Received: 24 May 2021

Accepted: 10 July 2021

Published: 16 July 2021

Publisher's Note: MDPI stays neutra with regard to jurisdictional claims in published maps and institutional affiliations.

Copyright: (c) 2021 by the authors. Licensee MDPI, Basel, Switzerland. This article is an open access article distributed under the terms and conditions of the Creative Commons Attribution (CC BY) license (https:// creativecommons.org/licenses/by/ $4.0 /)$.

\begin{abstract}
Given the inherent characteristics of nonlinearity and nonstationarity of iterative reconstruction algorithms in computed tomography (CT) imaging, this study aimed to perform, for the first time, a voxel-based characterization of noise properties in CT imaging with the ASiR-V and ASiR algorithms as compared with conventional filtered back projection (FBP). Multiple repeated scans of the Catphan-504 phantom were carried out. CT images were reconstructed using FBP and ASiR/ASiR-V with different blending levels of reconstruction $(20 \%, 40 \%, 60 \%, 80 \%, 100 \%)$. Noise maps and their nonuniformity index (NUI) were obtained according to the approach proposed by the report of AAPM TG-233. For the homogeneous CTP486 module, ASiR-V/ASiR allowed a noise reduction of up to $63.7 \% / 52.9 \%$ relative to FBP. While the noise reduction values of ASiR-V/ ASiR-reconstructed images ranged up to $33.8 \% / 39.9 \%$ and $31.2 \% / 35.5 \%$ for air and Teflon contrast objects, respectively, these values were approximately $60 \% / 50 \%$ for other contrast objects (PMP, LDPE, polystyrene, acrylic, Delrin). Moreover, for all contrast objects but air and Teflon, ASiR-V showed a greater noise reduction potential than ASiR when the blending level was $\geq 40 \%$. While noise maps of the homogenous CTP486 module showed only a slight spatial variation of noise (NUI $<5.2 \%$ ) for all reconstruction algorithms, the NUI values of iterative-reconstructed images of the nonhomogeneous CTP404 module increased nonlinearly with blending level and were 19\%/15\% and $6.7 \%$ for pure ASiR-V/ASiR and FBP, respectively. Overall, these results confirm the potential of ASiR-V and ASiR in reducing noise as compared with conventional FBP, suggesting, however, that the use of pure ASiR-V or ASiR might be suboptimal for specific clinical applications.
\end{abstract}

Keywords: computed tomography; iterative reconstruction algorithms; ASiR; ASiR-V; noise level; noise spatial uniformity

\section{Introduction}

The impact of computed tomography (CT) imaging on patient dose has been a topic of increasing attention in recent years [1-8]. Given that CT examinations are the major source of population exposure to ionizing radiation in industrialized countries [9,10], many strategies have been implemented to keep patient dose as low as reasonably achievable while maintaining a good diagnostic image quality [11]. In this regard, acquisition-related approaches, such as tube current modulation (both in the axial and longitudinal planes), appropriate selection of tube voltage according to patient size, table motion, and gantry rotation speed optimization have been successfully adopted on modern CT scanners [11-13]. 
The introduction of iterative reconstruction (IR) algorithms in clinical practice represents an important step toward dose optimization [13-18]. Unlike the conventional filtered backprojection (FBP) algorithm, IR algorithms take into account various physical aspects of CT image acquisition and can be based on statistical or model-based approaches [19-22]. While statistical-based IR algorithms usually consider the fluctuations in the projection measurement through a statistical method (e.g., by modeling the photon counting), model-based algorithms include a more complex description of physical and optical aspects in the reconstruction process, resulting in a better representation of the whole imaging process but in a longer reconstruction time [19-22]. When compared with conventional FBP, IR algorithms have proven to significantly decrease noise in CT imaging, allowing CT examinations with reduced patient exposure while providing still acceptable diagnostic images [23-28]. The noise properties of CT images obtained using IR algorithms can be rather different from those of FBP-derived CT images [20,21]. Indeed, IR algorithms can have an impact not only on the noise level but also on the noise texture of CT images [24,29-35]. Therefore, for each specific IR algorithm, a comprehensive characterization of the noise properties and quality of reconstructed CT images is recommended to adequately assess its dose reduction potential and usefulness.

So far, a number of IR algorithms with different characteristics have been implemented on clinical CT scanners [21,22], such as the adaptive statistical iterative reconstruction (ASiR) [36], adaptive statistical iterative reconstruction-V (ASiR-V) [37], and Veo [38] (GE Healthcare, Waukesha, WI, USA). The ASiR algorithm works on sinogram data by modeling the photon statistics and some noise-related properties of the scanned object (e.g., scatter), and can be employed with different FBP-ASiR blending levels [36]. Although ASiR is widely adopted due to its fast reconstruction time, some image quality properties, such as image texture and spatial resolution, depend on the blending level of reconstruction and the dose level, yielding some artificial image texture and degradation of spatial resolution at a higher percentage of blending for low-contrast objects $[24,29,33,39,40]$. The model-based Veo algorithm allows limiting these drawbacks by modeling the entire imaging system (i.e., X-ray tube output, detector response, radiation scatter properties, geometric features of the beam) $[21,22,34,41]$. However, due to the complexity of the imaging system model, Veo reconstruction time is greatly increased compared with ASiR, hampering its wide use in clinical practice $[31,34,41,42]$. The more recent ASiR-V algorithm has been proposed to reduce reconstruction time with respect to Veo while preserving some advantages of the full model-based approach in terms of image quality [22,34,36]. In particular, ASiR-V features more advanced noise and object modeling as compared with ASiR [36] and implements parts of the Veo physics model, but excludes the system optics in the modeling process, enabling reconstruction time almost comparable to that of FBP [36]. Like ASiR, ASiR-V can be blended with FBP at different percentages of reconstruction, from $0 \%$ (i.e., FBP) to $100 \%$ (i.e., pure ASiR-V) [36].

Several previous studies have characterized the image quality of CT imaging with ASiR $[24,28,29,33,39,43,44]$, finding substantial noise reduction properties as compared with conventional FBP. For instance, Miéville et al. showed that the benefit of ASiR in terms of noise reduction occurs almost independently of dose level (CTDI $\mathrm{vol}_{\mathrm{vol}}$ range: $\left.0.2-7.2 \mathrm{mGy}\right)$ with a noise reduction relative to FBP of approximately $20 \%$ and $50 \%$ for $\mathrm{ASiR} 40 \%$ and $\mathrm{ASiR} 100 \%$, respectively [24]. Analogously, Hussain et al. found, for two different radiation exposures (tube loads of 130 and $260 \mathrm{mAs}$ at $120 \mathrm{kVp}$ ), a noise reduction relative to FBP of approximately $30 \%$ and $50 \%$ with ASiR $50 \%$ and ASiR $100 \%$, respectively [43]. A study by Barca et al. confirmed that $\mathrm{ASiR}$ has the capability of reducing noise by up to $50 \%$ relative to FBP while preserving diagnostic information at low radiation exposure [33].

More recently, a number of clinical and phantom studies have evaluated the dose reduction potential and performance of ASiR-V [45-59]. In particular, some studies have assessed the noise properties of CT imaging with ASiR-V in comparison with FBP or other IR algorithms [45,46,49-55,57-59]. For instance, a clinical study by Gatti et al. showed that a relevant noise reduction can be obtained using $\mathrm{ASiR}-\mathrm{V} 40 \%$ with respect to $\mathrm{ASiR} 50 \%$ in 
the CT imaging of oncologic patients [50]. Goodenberger et al. compared abdomen and phantom CT images reconstructed by adopting FBP, ASiR, Veo, and ASiR-V algorithms [51]. They performed subjective and objective analyses showing that ASiR-V $30 \%$ and ASiR-V $60 \%$ provided the best combination of qualitative and quantitative performances. In a phantom study, Lim et al. compared the image quality of images reconstructed using FBP, ASiR, and ASiR-V algorithms [52]. They found that ASiR-V can allow an appreciable reduction of CT image noise and an improvement in contrast-to-noise ratio and spatial resolution. Euler et al. evaluated image noise, spatial resolution, lesion detectability, and dose reduction potential of $\mathrm{ASiR}-\mathrm{V}$ ( $50 \%$ and $100 \%)$ in comparison with FBP by performing phantom CT acquisitions [54]. They reported that ASiR-V has the capability of reducing image noise and improving low-contrast lesion detectability when compared with FBP. They suggested that such improvement in detection can allow radiation exposure reduction. De Marco and Origgi [55] assessed the noise level and noise power spectrum properties of $\mathrm{CT}$ imaging with ASiR-V, considering phantom images reconstructed with soft, standard, and bone kernels. As compared with FBP, they found a greater noise reduction provided by ASiR-V with respect to ASiR for soft and standard kernels, while less noise reduction was found for the bone kernel.

While these studies $[50-52,54,55]$ have explored in detail some image quality aspects of CT imaging with ASiR-V and possibly ASiR as compared with FBP, noise level has been estimated by computing the standard deviation (SD) of CT numbers within a given region of interest (ROI) $[17,60,61]$. However, this approach would be conceptually appropriate only for ergodic and stationary imaging systems, which feature uncorrelated noise [17,62]. Actually, due to image reconstruction and processing, CT image noise may present some degrees of correlation $[63,64]$. Moreover, this approach cannot provide information on the degree of spatial nonuniformity of noise [65]. Therefore, a more rigorous characterization of noise properties should be performed by computing voxelwise noise maps through multiple repeated CT imaging acquisitions $[17,41,65]$. Notably, these issues might be of particular relevance for IR algorithms given their inherent nonlinearity and nonstationarity properties. Therefore, the purpose of this phantom study was to carry out a voxel-based assessment of noise properties in CT imaging with ASiR-V and ASiR for different blending levels of reconstruction and contrast objects.

\section{Materials and Methods}

\subsection{Scanner and Phantom Acquisitions}

A 64-slice CT scanner (Optima CT660, GE Healthcare, Waukesha, WI, USA) was employed to acquire (helical scan mode; pitch, 0.984 ; rotation time, $1 \mathrm{~s}$; tube load, $100 \mathrm{mAs}$ without modulation; tube voltage, $120 \mathrm{kVp}$; collimation, $40 \mathrm{~mm}$ ) images of the Catphan-504 phantom (The Phantom Laboratory, Salem, NY, USA). The entire phantom was scanned 40 times under identical conditions. CT images (slice thickness, $2.5 \mathrm{~mm}$; display field of view, $250 \mathrm{~mm} \times 250 \mathrm{~mm}$; standard convolution kernel) were reconstructed using conventional FBP and $\mathrm{ASiR} / \mathrm{ASiR}-\mathrm{V}$ algorithms with different blending levels of reconstruction (20\%, $40 \%, 60 \%, 80 \%$, and $100 \%)$.

The Catphan-504 phantom has a cylindrical shape (20 cm outer diameter) and consists of four modules. In particular, the CTP486 and CTP404 modules were employed for noise properties assessment. The CTP486 module is a homogeneous medium, while the CTP404 module includes different contrast objects within a homogeneous background. Nominal CT numbers of the contrast objects are reported in Table 1. 
Table 1. Nominal CT numbers of the Catphan-CTP404 contrast objects [66].

\begin{tabular}{cc}
\hline Material & CT Numbers (HU) \\
\hline Air & $-1046:-986$ \\
PMP a & $-220:-172$ \\
LDPE $^{\text {b }}$ & $-121:-87$ \\
Polystyrene & $-65:-29$ \\
Acrylic & $92: 137$ \\
Delrin & $344: 387$ \\
Teflon & $941: 1060$ \\
\hline
\end{tabular}

a Polymethylpentene, ${ }^{\mathrm{b}}$ low-density polyethylene.

\subsection{Noise Maps}

In order to assess local noise properties, as suggested by the AAPM Task Group 233 report [65], noise maps of CT images reconstructed by using FBP (N-FBP), ASiR (N-ASiR), and ASiR-V (N-ASiR-V) were obtained on a voxel-by-voxel basis computing the SD of each voxel value across the 40 repeated acquisitions. Then, maps of the percentage difference between noise of FBP-reconstructed images and noise of both ASiR-V-reconstructed (PDN-ASiR-V) and ASiR-reconstructed (PDN-ASiR) images were computed voxelwise. In particular, N-FBP as well as N-ASiR, N-ASiR-V, PDN-ASiR-V, and PDN-ASiR maps with different blending levels of reconstruction were obtained for the slice at the central level of both the CTP486 and CTP404 phantom modules.

For CT imaging of the homogeneous CTP486 module, a summary noise level was estimated as the mean \pm SD value of N-FBP, N-ASiR, and N-ASiR-V maps within a central circular ROI of $1 \mathrm{~cm}$ diameter. Analogously, a summary percentage noise reduction of ASiR$\mathrm{V}$ and ASiR with respect to FBP was estimated as the mean \pm SD value of PDN-ASiR-V and PDN-ASiR maps, respectively, within a central circular ROI of $1 \mathrm{~cm}$ diameter.

For CT imaging of the CTP404 module, a summary noise value of each contrast object was estimated as the mean \pm SD value of N-FBP, N-ASiR, and N-ASiR-V maps within a circular ROI of $1 \mathrm{~cm}$ diameter placed in the center of the contrast object. Analogously, for each contrast object, a summary percentage noise reduction of ASiR-V and ASiR with respect to FBP was estimated as the mean \pm SD value of PDN-ASiR-V and PDN-ASiR maps, respectively, within a circular ROI of $1 \mathrm{~cm}$ diameter placed in the center of the contrast object.

In order to assess the spatial nonuniformity degree of N-FBP, N-ASiR, and N-ASiR$\mathrm{V}$ maps, the nonuniformity index (NUI) [67], as proposed by the AAPM Task Group 233 report [65], was employed. Specifically, for both the CTP486 and CTP404 modules, the entire inner part of the phantom (diameter, $15 \mathrm{~cm}$ ) was divided into $k=249$ ROIs (size, $8 \mathrm{~mm} \times 8 \mathrm{~mm}$ ). Then, for each map (N-FBP, N-ASiR, and N-ASiR-V), the NUI was calculated as:

$$
N U I=\frac{100}{<m>} \sqrt{\frac{1}{k-1} \sum_{i=1}^{k}\left(m_{i}-<m>\right)^{2}}
$$

where $m_{i}$ and $\langle m>$ are the average of the values of the considered map within the $i$-th ROI and the average of all $m_{i}$ values, respectively.

Furthermore, in order to characterize the spatial distribution of noise in CT imaging with $\mathrm{ASiR}$ and ASiR-V reconstruction algorithms, the histograms of N-ASiR and N-ASiR-V maps, as well as of N-FBP map, were computed for the entire inner part of the CTP486 module (diameter, $15 \mathrm{~cm}$ ). Then, the histogram descriptors of median, interquartile range, kurtosis, and skewness were calculated.

Image analysis was performed by using the MATLAB R2018a (MathWorks, Natick, MA, USA) software package. 


\section{Results}

$\mathrm{N}-\mathrm{FBP}$ and N-ASiR/N-ASiR-V maps (with different blending levels of reconstruction) of the CTP486 homogeneous module and CTP404 module with contrast objects are shown in Figures 1 and 2, respectively.



Figure 1. Noise maps (HU) of a CT image of the CTP486 phantom module reconstructed using FBP and $\mathrm{ASiR} / \mathrm{ASiR}-\mathrm{V}$ with different blending levels $(20 \%, 40 \%, 60 \%, 80 \%$, and $100 \%)$. The corresponding FBP-reconstructed CT image of the phantom is also displayed.



Figure 2. Noise maps (HU) of a CT image of the CTP404 phantom module reconstructed using FBP and ASiR/ASiR-V with different blending levels $(20 \%, 40 \%, 60 \%, 80 \%$, and $100 \%)$. The corresponding FBP-reconstructed CT image of the phantom is also displayed.

Moreover, PDN-ASiR and PDN-ASiR-V maps (with different blending levels of reconstruction) of the CTP486 homogeneous module and CTP404 module with contrast objects are displayed in Figures 3 and 4, respectively.



Figure 3. CTP486 phantom module. Maps of the percentage difference (\%) between noise of the FBP-reconstructed CT image and noise of both ASiR-V-reconstructed and ASiR-reconstructed CT images with different blending levels $(20 \%, 40 \%, 60 \%, 80 \%$, and $100 \%)$.

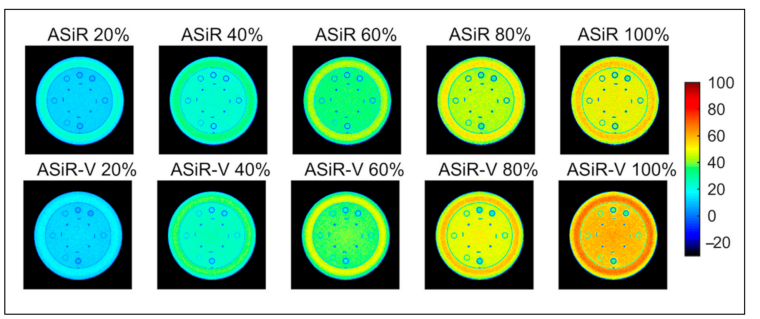

Figure 4. CTP404 phantom module. Maps of the percentage difference (\%) between noise of the FBP-reconstructed CT image and noise of both ASiR-V-reconstructed and ASiR-reconstructed CT images with different blending levels $(20 \%, 40 \%, 60 \%, 80 \%$, and $100 \%)$. 
Noise maps of the homogenous CTP486 phantom module (Figure 1) showed only a slight spatial variation of local noise values for the FBP-reconstructed CT image, which seemed less for ASiR-V- and ASiR-reconstructed CT images with higher blending levels. This is corroborated by the NUI values reported in detail in Figure 5. In particular, the NUI value for the FBP-reconstructed CT image was $5.2 \%$, while the NUI values for the ASiR-V- and ASiR-reconstructed CT images with a $100 \%$ blending level were $2.7 \%$ and $3.4 \%$, respectively. On the other hand, noise maps of the CTP404 phantom module (including various contrast objects) (Figure 2) showed a more appreciable degree of spatial variation of noise, with higher noise values at the boundary between each contrast object and background, especially for ASiR-V- and ASiR-reconstructed CT images. The NUI value for the FBP-reconstructed CT image was 6.7\%. The NUI values for the IR-reconstructed CT images increased nonlinearly with the blending level and were $19 \%$ and $15 \%$ for pure $\mathrm{ASiR}-\mathrm{V}$ and ASiR, respectively.

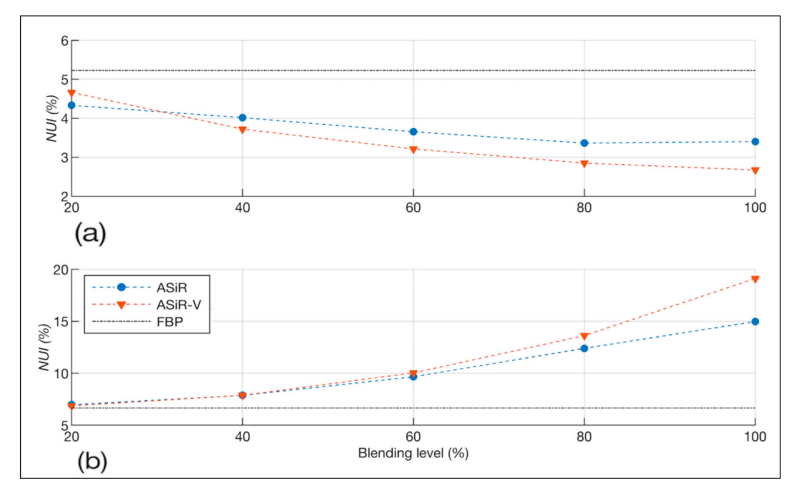

Figure 5. Nonuniformity index (NUI) values of noise maps of CT reconstructed images (FBP, ASiR-V, and ASiR) of the CTP486 (a) and CTP404 (b) phantom modules. For NUI estimation, only the inner part of the phantom (diameter, $15 \mathrm{~cm}$ ) was considered.

As shown by PDN-ASiR-V and PDN-ASiR maps of the homogenous CTP486 module (Figure 3), when compared with conventional FBP, both ASiR and ASiR-V allowed a substantial noise reduction in CT imaging. In this regard, noise values in the CT imaging of the homogenous CTP486 phantom module are reported in Table 2 for FBP, ASiR-V, and ASiR. The percentage difference between noise in the FBP-reconstructed CT image and that in both ASiR-V- and ASiR-reconstructed CT images increased with increasing blending level, up to $64 \%$ and $53 \%$ for $\mathrm{ASiR}-\mathrm{V}$ and $\mathrm{ASiR}$, respectively (Figure 6). Except for the lowest blending level of $20 \%$, ASiR-V presented greater noise reduction potential than ASiR (Figure 6).

Table 2. Homogeneous CTP486 module. Noise level estimated as the mean (SD) value of N-FBP, $\mathrm{N}-\mathrm{ASiR}$, and N-ASiR-V maps within a central circular ROI of $1 \mathrm{~cm}$ diameter.

\begin{tabular}{cccc}
\hline Blending Level & FBP & ASiR & ASiR-V \\
\hline $0 \%$ & $9.5(1.3)$ & & \\
$20 \%$ & & $7.9(1.1)$ & $8.0(1.2)$ \\
$40 \%$ & & $6.9(1.0)$ & $6.5(1.0)$ \\
$60 \%$ & $5.8(0.8)$ & $5.4(0.8)$ \\
$80 \%$ & & $4.9(0.7)$ & $4.4(0.6)$ \\
$100 \%$ & & $4.5(0.7)$ & $3.4(0.5)$ \\
\hline
\end{tabular}




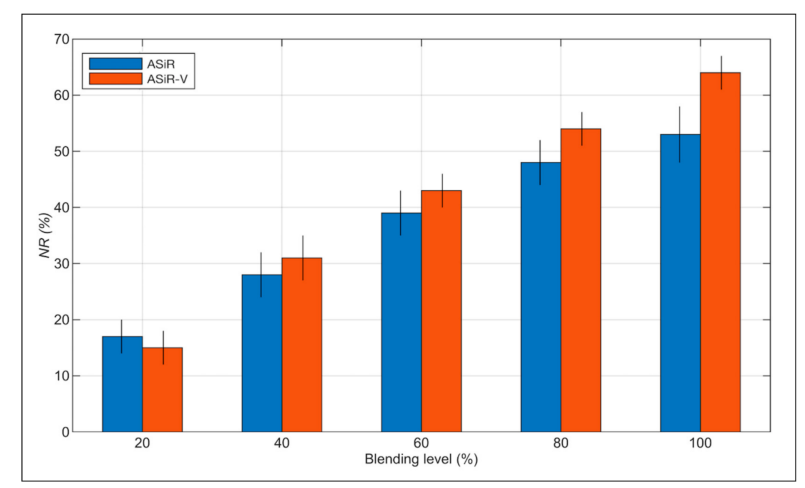

Figure 6. CTP486 homogeneous phantom module. Noise reduction properties of ASiR-V and ASiR reconstruction algorithms with respect to conventional FBP, $N R(\%)$, estimated as the mean (SD) value of PDN-ASiR-V and PDN-ASiR-V maps within a central circular ROI of $1 \mathrm{~cm}$ diameter.

For different contrast objects (i.e., air, PMP, LDPE, polystyrene, acrylic, Delrin, Teflon) of the CTP404 phantom module, summary noise level values in CT imaging using FBP and $\mathrm{ASiR}-\mathrm{V} / \mathrm{ASiR}$ with different blending levels of reconstruction are reported in detail in Table 3. Figure 7 shows the noise reduction properties of $A S i R-V$ and ASiR reconstruction algorithms with respect to conventional FBP for different contrast objects and blending levels of reconstruction. For all contrast objects, when compared with conventional FBP, both ASiR-V and ASiR allowed a substantial reduction in noise level, which decreased with an increasing blending level of reconstruction (Table 3). The noise reduction properties of both ASiR and ASiR-V depended on the contrast object. In particular, higher contrast objects (i.e., air and Teflon) were characterized by a lower noise reduction degree (Figure 7). Moreover, for all contrast objects but air and Teflon, ASiR-V showed a greater noise reduction potential than $\mathrm{ASiR}$ when the blending level was $\geq 40 \%$.

Table 3. CTP404 phantom module with different contrast objects (i.e., air, PMP, LDPE, polystyrene, acrylic, Delrin, Teflon). Summary noise level of each contrast object estimated as the mean (SD) value of N-FBP, N-ASiR, and N-ASiR-V maps within a central circular ROI of $1 \mathrm{~cm}$ diameter.

\begin{tabular}{ccccccccc}
\hline & & Air & PMP & LDPE & Polystyrene & Acrylic & Delrin & Teflon \\
\hline FBP & & $9.0(1.0)$ & $9.7(1.1)$ & $9.8(1.1)$ & $9.6(1.0)$ & $9.7(1.2)$ & $10.4(1.1)$ & $11.3(1.3)$ \\
\hline \multirow{6}{*}{ ASiR } & $20 \%$ & $8.1(0.9)$ & $8.6(0.9)$ & $8.6(0.9)$ & $8.4(0.9)$ & $8.5(1.0)$ & $9.2(0.9)$ & $10.2(1.2)$ \\
& $40 \%$ & $7.3(0.8)$ & $7.5(0.8)$ & $7.6(0.8)$ & $7.4(0.8)$ & $7.5(0.9)$ & $8.1(0.8)$ & $9.4(1.1)$ \\
& $60 \%$ & $6.6(0.8)$ & $6.5(0.7)$ & $6.5(0.7)$ & $6.4(0.7)$ & $6.4(0.8)$ & $7.0(0.7)$ & $8.5(1.1)$ \\
& $100 \%$ & $5.4(0.7)$ & $5.1(0.7)$ & $5.0(0.6)$ & $4.9(0.6)$ & $5.0(0.7)$ & $5.5(0.7)$ & $7.3(1.0)$ \\
\hline \multirow{4}{*}{ ASiR- } & $40 \%$ & $8.4(0.9)$ & $8.5(0.9)$ & $8.6(0.9)$ & $8.5(0.9)$ & $8.5(1.0)$ & $9.1(0.9)$ & $10.5(1.2)$ \\
V & $60 \%$ & $7.1(0.9)$ & $6.3(0.7)$ & $6.3(0.7)$ & $6.2(0.7)$ & $6.2(0.7)$ & $6.7(0.7)$ & $9.0(1.2)$ \\
& $80 \%$ & $6.5(0.9)$ & $5.3(0.6)$ & $5.2(0.6)$ & $5.2(0.6)$ & $5.1(0.6)$ & $5.6(0.6)$ & $8.3(1.2)$ \\
& $100 \%$ & $6.0(1.0)$ & $4.3(0.5)$ & $4.2(0.5)$ & $4.2(0.5)$ & $4.1(0.5)$ & $4.6(0.5)$ & $7.7(1.3)$ \\
\hline
\end{tabular}

Histograms of N-ASiR and N-ASiR-V maps, as well as of the N-FBP map, are shown in Figure 8. Moreover, values of histogram descriptors (median, interquartile range, kurtosis, skewness) are reported in detail in Table 4. While noise map histogram of the FBP-reconstructed CT image approximated a Gaussian distribution (kurtosis and skewness values of 3 and 0.23 , respectively), noise map histograms of ASiR- and ASiR-Vreconstructed CT images leaned to deviate from a Gaussian distribution when increasing the blending level of reconstruction, with kurtosis/skewness values for pure ASiR- and ASiR-V-reconstructed CT images of 3.57/0.41 and 3.35/0.24, respectively. 


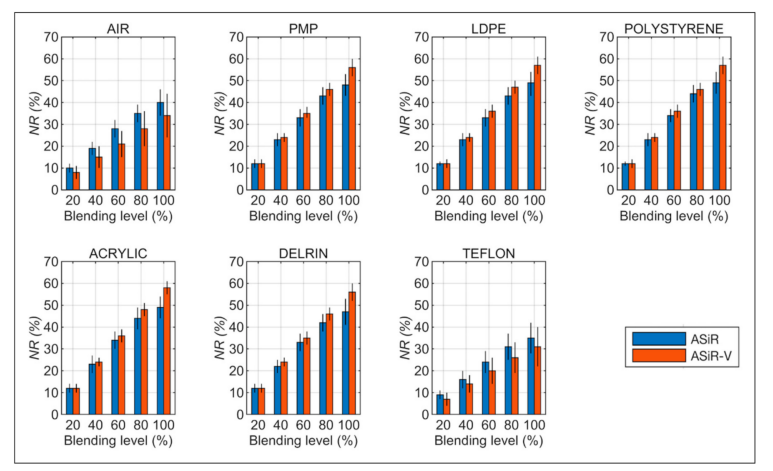

Figure 7. CTP404 phantom module with different contrast objects (i.e., air, PMP, LDPE, polystyrene, acrylic, Delrin, Teflon). Noise reduction properties of $\mathrm{ASiR}-\mathrm{V}$ and $\mathrm{ASiR}$ reconstruction algorithms with respect to conventional FBP, $N R(\%)$, estimated as the mean (SD) value of PDN-ASiR-V and PDN-ASiR-V maps within a central circular ROI of $1 \mathrm{~cm}$ diameter placed in each contrast object.

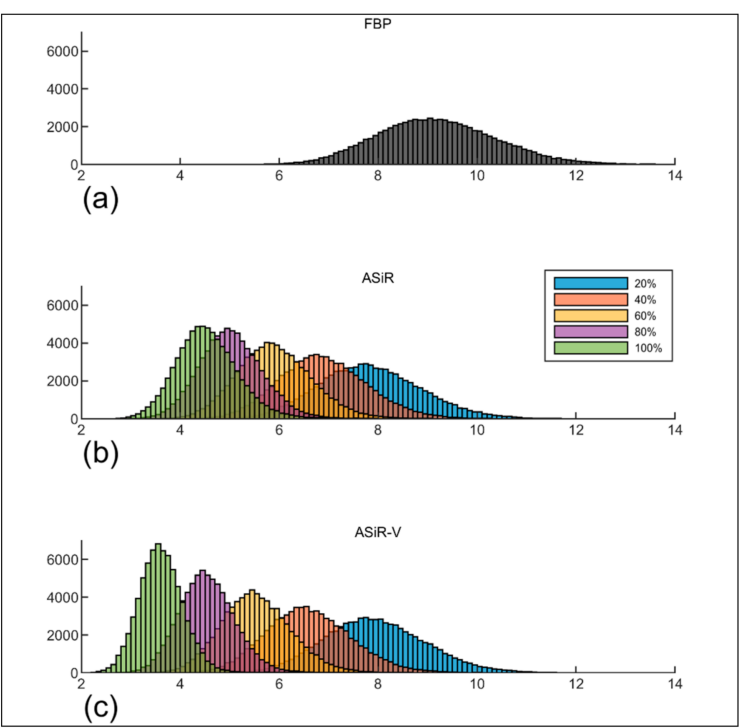

Figure 8. Histograms of noise maps (bin width of $0.1 \mathrm{HU}$ ) for FBP- (a), ASiR- (b), and ASiR-Vreconstructed (c) CT images of the homogenous CTP486 module with different blending levels of reconstruction $(20 \%, 40 \%, 60 \%, 80 \%$, and $100 \%)$.

Table 4. Descriptive metrics of noise map histograms for FBP-, ASiR-, and ASiR-V-reconstructed CT images of the homogenous CTP486 module with different blending levels of reconstruction $(20 \%$, $40 \%, 60 \%, 80 \%$, and $100 \%$ ). Median and interquartile range values are expressed as HU; kurtosis and skewness values are unitless.

\begin{tabular}{cccccc}
\hline & Median & $\begin{array}{c}\text { Interquartile } \\
\text { Range }\end{array}$ & Kurtosis & Skewness \\
\hline FBP & & 9.16 & 1.62 & 3.00 & 0.23 \\
\hline \multirow{3}{*}{ ASiR } & $20 \%$ & 7.81 & 1.39 & 3.07 & 0.27 \\
& $40 \%$ & 6.86 & 1.17 & 3.10 & 0.26 \\
& $60 \%$ & 5.88 & 0.99 & 3.13 & 0.25 \\
& $80 \%$ & 5.00 & 0.83 & 3.14 & 0.22 \\
ASiR-V & $100 \%$ & 4.51 & 0.80 & 3.57 & 0.41 \\
& $20 \%$ & 7.91 & 1.37 & 3.03 & 0.24 \\
& $40 \%$ & 6.57 & 1.15 & 3.10 & 0.27 \\
& $60 \%$ & 5.51 & 0.92 & 3.11 & 0.25 \\
& $80 \%$ & 4.52 & 0.74 & 3.14 & 0.23 \\
& $100 \%$ & 3.60 & 0.57 & 3.35 & 0.24 \\
\hline
\end{tabular}




\section{Discussion}

Given that IR algorithms in CT imaging can be inherently nonlinear and nonstationary, noise properties should be possibly assessed by using a rigorous voxel-based approach exploiting multiple repeated acquisitions. Unlike the widely employed approach of estimating noise as the SD of CT numbers within an ROI, this more complex approach allows for solving potential issues related to nonvalidity of the ergodic theorem for CT imaging with IRs, as well as for assessing the spatial nonuniformity degree of noise. However, so far, only few studies [67-70] have employed such an approach to characterize noise properties in CT imaging with IRs. In particular, Solomon and Samei [68] performed multiple repeated CT acquisitions of a uniform phantom and of two anatomically informed textured phantoms, and used these phantoms to assess noise properties for FBP and the sinogram affirmed iterative reconstruction (SAFIRE, Siemens Healthcare) algorithm. They found that noise was globally nonstationary, and this was mostly relevant for SAFIRE-reconstructed CT images of textured phantoms. In a similar study, Solomon et al. [69] showed that the advanced modeled iterative reconstruction (ADMIRE, Siemens Healthcare) algorithm with different iterative strengths (i.e., 3, 4, and 5) allowed a noise reduction of up to $64 \%$ relative to FBP. A study by Funama et al. [70] compared noise in CT images of a porcine liver phantom surrounded by agar gel acquired 100 times, reconstructed using FBP and the iterative model reconstruction (IMR, Philips Healthcare) algorithm with various noise reduction levels (L1, L2, and L3, where L3 offers the greatest noise reduction). They revealed a spatial dependence of noise for IMR-reconstructed CT images. Moreover, for IMR-L3, a noise reduction of $68 \%-75 \%$ relative to FBP was observed. Li et al. [67] acquired 50 repeated acquisitions of a homogeneous phantom in order to assess the spatial nonuniformity of noise maps associated with FBP- and Veo-reconstructed images. Veo-reconstructed CT images were characterized by at least a $50 \%$ reduction in NUI values relative to FBP-reconstructed CT images.

Some previous clinical or phantom studies have reported a higher noise reduction capability of ASiR-V as compared with ASiR. For instance, Gatti et al. [50] conducted a retrospective case-control study involving oncologic patients who underwent baseline and follow-up CT scans with ASiR 50\% and ASiR-V 40\%, respectively. Noise was estimated as the SD of CT numbers within three circular ROIs placed in homogeneous regions of three different tissues. Regardless of the anatomical region, noise was found lower in ASiR-V 40\%-reconstructed images than in ASiR 50\%-reconstructed images, albeit radiation exposure was 38\% lower for the ASIR-V examinations than for the ASiR ones. Similarly, Goodenberger et al. carried out a retrospective study on 36 patients who underwent an abdomino-pelvic CT scan [51]. They assessed the noise level within lesions for CT images reconstructed using FBP, ASiR 80\%, Veo, and ASiR-V (30\%, 60\%, and 90\%). The highest noise level was revealed on FBP-reconstructed images, followed by ASiR-V 30\%-, ASiR 80\%-, Veo-, ASiR-V 60\%-, and ASiR-V 90\%-reconstructed images. Lim et al. [52] compared the noise performances of $\mathrm{ASiR}-\mathrm{V}, \mathrm{ASiR}$, and $\mathrm{FBP}$ on an anthropomorphic phantom using three blending levels of reconstruction $(30 \%, 50 \%$, and $70 \%)$ and five different tube currents. By assessing the noise magnitude and noise reduction relative to FBP within a circular ROI in a homogeneous area of the phantom, they found that ASiR-V 30\%, ASiR-V 50\%, and ASiR-V $70 \%$ can allow a noise reduction of $30 \%-45 \%, 41 \%-61 \%$, and $52 \%-67 \%$, respectively. On the other hand, ASiR with the same blending levels showed a lower noise reduction of $18 \%-20 \%, 30 \%-33 \%$, and $41 \%-49 \%$, respectively. Euler et al. [54] characterized the noise level of ASiR-V-reconstructed (50\% and $100 \%$ blending levels of reconstruction) CT images. On average, they found a noise reduction of 37\% (ASiR-V 50\%) and 72\% (ASiR-V 100\%) relative to FBP. Another phantom study by De Marco and Origgi [55] compared the noise levels of FBP-, ASiR-V-, and ASiR-reconstructed images (blending levels of $40 \%, 60 \%$, and $100 \%$ ) acquired on two different CT scanners (i.e., ASiR-V- and ASiR-reconstructed images were not obtained from the same sinogram data). Specifically, this study assessed the effect of convolution kernel (standard, soft, and bone), slice thickness, and radiation exposure on noise level for all the used reconstruction algorithms. When compared with FBP, ASiR-V 
showed a greater noise (estimated as the SD of CT numbers within a square ROI in a homogeneous phantom) reduction than ASiR with noise reduction values of $12 \%-28 \%$, $18 \%-41 \%$, and $29 \%-64 \%$ for ASiR-V $40 \%, 60 \%$, and $100 \%$, respectively. For both ASiR-V and $\mathrm{ASiR}$, noise reduction relative to FBP was almost independent of dose exposure.

To the best of our knowledge, this is the first study that performed, for different blending levels of reconstruction and contrast objects, a voxel-based assessment of noise properties in $\mathrm{CT}$ imaging with $\mathrm{ASiR}-\mathrm{V}$ and $\mathrm{ASiR}$ algorithms as compared with conventional FBP. This approach, which can allow a better characterization of noise properties in CT imaging with IR algorithms, is based on spatial noise maps (Figures 1 and 2). In general, we found that, when compared with FBP, ASiR-V presents a greater noise reduction than ASiR for a homogeneous medium, except for the lowest blending level of 20\% (Figure 6). In particular, the noise reduction value ranged up to $52.9 \%$ and $63.7 \%$ for pure ASiR and pure ASiR-V, respectively. This finding has also been confirmed for various contrast objects (i.e., PMP, LDPE, polystyrene, acrylic, Delrin) (Figure 7) with noise reduction values rather independent of contrast objects and ranging up to approximately $50 \%$ and $60 \%$ for pure $\mathrm{ASiR}$ and pure ASiR-V, respectively. On the other hand, for higher contrast objects (i.e., air and Teflon) and all blending levels, ASiR-V showed a lower noise reduction capability than $\mathrm{ASiR}$. In this case, the noise reduction values of $\mathrm{ASiR}-\mathrm{V}-/ \mathrm{ASiR}$-reconstructed images were lower and ranged up to $33.8 \% / 39.9 \%$ and $31.2 \% / 35.5 \%$ for air and Teflon contrast objects, respectively.

In this work, unlike a few previous studies on the characterization of noise in CT imaging with ASiR-V and ASiR [50-52,54,55], voxelwise noise maps allow an assessment of the spatial uniformity of noise, which might be affected by the nonlinearity and nonstationarity effects of IR algorithms. For the homogeneous medium, FBP-reconstructed images showed a rather low level of the spatial nonuniformity of noise with an NUI value of $5.2 \%$. The NUI values of ASiR-V- and ASiR-reconstructed CT images were lower than the NUI value of FBP-reconstructed images and slightly decreased with an increasing blending level of reconstruction (Figure 5a). This could be partly associated with a shift of noise power spectrum curves toward lower spatial frequencies, which has been observed for CT imaging with ASiR-V and ASiR mostly at higher blending levels of reconstruction $[24,29,35,55]$. Notably, for the nonhomogeneous medium with contrast objects, both ASiR-V- and ASiR-reconstructed CT images showed NUI values greater than the NUI value of FBP-reconstructed CT images (approximately 7\%), increasing with the blending level of reconstruction (Figure 5b). In particular, the NUI values of CT imaging with pure ASiR-V and pure ASiR were approximately three and two times, respectively, that of CT imaging with FBP. This is likely to derive from a noise reduction capability of ASiR-V and ASiR reconstruction algorithms that does not seem so effective at the boundary between different media (Figures 2 and 4). While this effect has been reported for other IR algorithms [68-70], no previous study has observed it for CT imaging with ASiR-V and ASiR. As submitted by Solomon and Samei [68], given that IR algorithms aim at reducing noise while preserving the fidelity of fine details, this necessitates a conservative approach to noise reduction for voxels near edges, and therefore, noise is not reduced as much near edges or structures (i.e., "edge" effect).

The nonuniformity findings of this study suggest that, for CT imaging, a noise evaluation only in homogeneous phantoms could be incomplete and/or partly misleading when IR algorithms are employed. While pure ASiR-V/ASiR offers the maximum noise reduction capability in reconstructed CT images, a blending level of $100 \%$ can affect the spatial uniformity of noise mostly at the boundary between different structures. Therefore, for specific clinical applications of CT imaging with ASiR-V and ASiR, the use of a maximum blending level of reconstruction might be not optimal [27,40,43,44,71,72]. In particular, the revealed "edge" effect might partly limit the potential of IR algorithms in studying small structures (such as metastases, vessels, pulmonary nodules, or brain ischemic lesions), especially when they present reduced contrast. 
Analysis of noise map histograms (Table 4) showed that, for both ASiR- and ASiR-Vreconstructed $\mathrm{CT}$ images, kurtosis and interquartile range/median values monotonically increased and decreased, respectively, with increasing blending level of reconstruction. Moreover, for all the blending levels of reconstruction, noise map histograms of ASiRreconstructed $\mathrm{CT}$ images were characterized by higher or equal kurtosis and interquartile range values compared with that of ASiR-V-reconstructed CT images. Overall, these results might reflect the inherent nonlinear and nonstationary properties of iterative reconstruction algorithms in CT imaging.

\section{Conclusions}

In this preliminary study, we aimed at characterizing in greater detail noise properties in CT imaging with ASiR-V and ASiR by means of spatial noise maps obtained from multiple repeated acquisitions of a homogeneous phantom and of a phantom with different contrast objects. Our results confirm the potential of ASiR-V and ASiR in reducing noise (up to $60 \%$ or more) as compared with conventional FBP. In this regard, while ASiR-V outperforms ASiR in general, this does not necessarily hold true for the CT imaging of structures with high contrast. Both ASiR-V- and ASiR-reconstructed CT images show a higher spatial nonuniformity of noise than FBP-reconstructed images. This effect increases with increasing blending level of reconstruction and reflects a less effective noise reduction potential of ASiR-V and ASiR at the boundary between different structures.

In summary, an exhaustive characterization of noise properties in CT imaging with IR algorithms is needed to actually assess the dose reduction potential of this technique and its possible limitations for specific clinical applications. This study suggests that, when using IR algorithms, the conventional approach of estimating the noise level by computing the $\mathrm{SD}$ of $\mathrm{CT}$ numbers within a given $\mathrm{ROI}$ is inadequate and precludes showing any non-negligible spatial nonuniformity of noise, which may vary with the blending level of reconstruction. Overall, our findings indicate that a voxel-based characterization of noise properties is recommended when dealing with a novel IR algorithm or when comparing the performances of different IR algorithms.

Author Contributions: Conceptualization, P.B. and M.G.; methodology, P.B., D.M. and M.G.; software, D.M.; validation, P.B., D.M., and M.G.; formal analysis, D.M.; investigation, P.B., D.M., and M.G.; resources, A.C.T. and M.G.; data curation, P.B. and D.M.; writing-original draft preparation, P.B., D.M., and M.G.; writing-review and editing, P.B., D.M., C.M., S.C., S.D., A.C.T. and M.G.; visualization, P.B., D.M., C.M., S.C., S.D., A.C.T. and M.G.; supervision, M.G.; project administration, M.G. All authors have read and agreed to the published version of the manuscript.

Funding: This research received no external funding.

Data Availability Statement: The data presented in this study are available on reasonable request from the corresponding author.

Acknowledgments: The authors would like to thank Davide Caramella for a stimulating and constructive discussion.

Conflicts of Interest: The authors declare no conflict of interest.

\section{References}

1. Brenner, D.J.; Hall, E.J. Computed Tomography-An Increasing Source of Radiation Exposure. N. Engl. J. Med. 2007, 357, 2277-2284. [CrossRef] [PubMed]

2. Boone, J.M.; Brink, J.A.; Edyvean, S.; Huda, W.; Leitz, W.; McCollough, C.H.; McNitt-Gray, M.F. Report 87. J. ICRU 2012 , 12. [CrossRef]

3. Thrall, J.H. Radiation Exposure in CT Scanning and Risk: Where Are We? Radiology 2012, 264, 325-328. [CrossRef] [PubMed]

4. Smith-Bindman, R.; Wang, Y.; Chu, P.; Chung, R.; Einstein, A.J.; Balcombe, J.; Cocker, M.; Das, M.; Delman, B.N.; Flynn, M.; et al. International Variation in Radiation Dose for Computed Tomography Examinations: Prospective Cohort Study. BMJ $2019, \mathrm{k} 4931$. [CrossRef]

5. Lumbreras, B.; Salinas, J.M.; Gonzalez-Alvarez, I. Cumulative Exposure to Ionising Radiation from Diagnostic Imaging Tests: A 12-Year Follow-up Population-Based Analysis in Spain. BMJ Open 2019, 9, e030905. [CrossRef] [PubMed] 
6. Andersson, J.; Pavlicek, W.; Al-Senan, R.; Cody, D.; Dixon, R.; Columbo, P.; Dong, F.; Edyvean, S.; Jansen, J.; Kanal, K.; et al. Estimating Patient Organ Dose with Computed Tomography: A Review of Present Methodology and Required DICOM Information. A Joint Report of AAPM Task Group 246 and the European Federation of Organizations for Medical Physics (EFOMP); AAPM: Alexandria, VA, USA, 2019.

7. Rehani, M.M.; Yang, K.; Melick, E.R.; Heil, J.; Šalát, D.; Sensakovic, W.F.; Liu, B. Patients Undergoing Recurrent CT Scans: Assessing the Magnitude. Eur. Radiol. 2020, 30, 1828-1836. [CrossRef]

8. De Mattia, C.; Campanaro, F.; Rottoli, F.; Colombo, P.E.; Pola, A.; Vanzulli, A.; Torresin, A. Patient Organ and Effective Dose Estimation in CT: Comparison of Four Software Applications. Eur. Radiol. Exp. 2020, 4, 14. [CrossRef]

9. European Commission; Directorate-General for Energy. Medical Radiation Exposure of the European Population; Radiation Protection; Publications Office of the European Union: Luxembourg, 2015; ISBN 978-92-79-45374-8.

10. National Council on Radiation Protection and Measurements (Ed.) Medical Radiation Exposure of Patients in the United States: Recommendations of the National Council on Radiation Protection and Measurements; NCRP Report; National Council on Radiation Protection and Measurements: Bethesda, MD, USA, 2019; ISBN 978-1-944888-16-9.

11. McNitt-Gray, M.F. AAPM/RSNA Physics Tutorial for Residents. Topics in CT: Radiation Dose in CT. RadioGraphics 2002, 22, 1541-1553. [CrossRef]

12. Gunn, M.L.D.; Kohr, J.R. State of the Art: Technologies for Computed Tomography Dose Reduction. Emerg. Radiol. 2010, 17, 209-218. [CrossRef]

13. Silva, A.C.; Lawder, H.J.; Hara, A.; Kujak, J.; Pavlicek, W. Innovations in CT Dose Reduction Strategy: Application of the Adaptive Statistical Iterative Reconstruction Algorithm. Am. J. Roentgenol. 2010, 194, 191-199. [CrossRef] [PubMed]

14. Costello, J.E.; Cecava, N.D.; Tucker, J.E.; Bau, J.L. CT Radiation Dose: Current Controversies and Dose Reduction Strategies. Am. J. Roentgenol. 2013, 201, 1283-1290. [CrossRef]

15. Hara, A.K.; Paden, R.G.; Silva, A.C.; Kujak, J.L.; Lawder, H.J.; Pavlicek, W. Iterative Reconstruction Technique for Reducing Body Radiation Dose at CT: Feasibility Study. Am. J. Roentgenol. 2009, 193, 764-771. [CrossRef]

16. Kalra, M.K.; Woisetschläger, M.; Dahlström, N.; Singh, S.; Lindblom, M.; Choy, G.; Quick, P.; Schmidt, B.; Sedlmair, M.; Blake, M.A.; et al. Radiation Dose Reduction with Sinogram Affirmed Iterative Reconstruction Technique for Abdominal Computed Tomography. J. Comput. Assist. Tomogr. 2012, 36, 339-346. [CrossRef] [PubMed]

17. Vaishnav, J.Y.; Jung, W.C.; Popescu, L.M.; Zeng, R.; Myers, K.J. Objective Assessment of Image Quality and Dose Reduction in CT Iterative Reconstruction: Validating Dose Reduction Claims for CT Iterative Reconstruction. Med. Phys. 2014, $41,071904$. [CrossRef]

18. Padole, A.; Ali Khawaja, R.D.; Kalra, M.K.; Singh, S. CT Radiation Dose and Iterative Reconstruction Techniques. Am. J. Roentgenol. 2015, 204, W384-W392. [CrossRef]

19. Beister, M.; Kolditz, D.; Kalender, W.A. Iterative Reconstruction Methods in X-Ray CT. Phys. Med. 2012, 28, 94-108. [CrossRef]

20. Willemink, M.J.; de Jong, P.A.; Leiner, T.; de Heer, L.M.; Nievelstein, R.A.J.; Budde, R.P.J.; Schilham, A.M.R. Iterative Reconstruction Techniques for Computed Tomography Part 1: Technical Principles. Eur. Radiol. 2013, 23, 1623-1631. [CrossRef]

21. Geyer, L.L.; Schoepf, U.J.; Meinel, F.G.; Nance, J.W.; Bastarrika, G.; Leipsic, J.A.; Paul, N.S.; Rengo, M.; Laghi, A.; De Cecco, C.N. State of the Art: Iterative CT Reconstruction Techniques. Radiology 2015, 276, 339-357. [CrossRef]

22. Stiller, W. Basics of Iterative Reconstruction Methods in Computed Tomography: A Vendor-Independent Overview. Eur. J. Radiol. 2018, 109, 147-154. [CrossRef]

23. Pickhardt, P.J.; Lubner, M.G.; Kim, D.H.; Tang, J.; Ruma, J.A.; del Rio, A.M.; Chen, G.-H. Abdominal CT with Model-Based Iterative Reconstruction (MBIR): Initial Results of a Prospective Trial Comparing Ultralow-Dose with Standard-Dose Imaging. Am. J. Roentgenol. 2012, 199, 1266-1274. [CrossRef]

24. Miéville, F.A.; Gudinchet, F.; Brunelle, F.; Bochud, F.O.; Verdun, F.R. Iterative Reconstruction Methods in Two Different MDCT Scanners: Physical Metrics and 4-Alternative Forced-Choice Detectability Experiments-A Phantom Approach. Phys. Med. 2013, 29, 99-110. [CrossRef]

25. Willemink, M.J.; Leiner, T.; de Jong, P.A.; de Heer, L.M.; Nievelstein, R.A.J.; Schilham, A.M.R.; Budde, R.P.J. Iterative Reconstruction Techniques for Computed Tomography Part 2: Initial Results in Dose Reduction and Image Quality. Eur. Radiol. 2013, 23, 1632-1642. [CrossRef]

26. Chen, J.-H.; Jin, E.-H.; He, W.; Zhao, L.-Q. Combining Automatic Tube Current Modulation with Adaptive Statistical Iterative Reconstruction for Low-Dose Chest CT Screening. PLoS ONE 2014, 9, e92414. [CrossRef]

27. Smith, E.A.; Dillman, J.R.; Goodsitt, M.M.; Christodoulou, E.G.; Keshavarzi, N.; Strouse, P.J. Model-Based Iterative Reconstruction: Effect on Patient Radiation Dose and Image Quality in Pediatric Body CT. Radiology 2014, 270, 526-534. [CrossRef] [PubMed]

28. Mangat, J.; Morgan, J.; Benson, E.; Båth, M.; Lewis, M.; Reilly, A. A Study of the Image Quality of Computed Tomography Adaptive Statistical Iterative Reconstructed Brain Images Using Subjective and Objective Methods. Radiat. Prot. Dosim. 2016, 169, 92-99. [CrossRef] [PubMed]

29. Samei, E.; Richard, S. Assessment of the Dose Reduction Potential of a Model-Based Iterative Reconstruction Algorithm Using a Task-Based Performance Metrology. Med. Phys. 2014, 42, 314-323. [CrossRef] [PubMed]

30. Dodge, C.T.; Tamm, E.P.; Cody, D.D.; Liu, X.; Jensen, C.T.; Wei, W.; Kundra, V.; Rong, X.J. Performance Evaluation of Iterative Reconstruction Algorithms for Achieving CT Radiation Dose Reduction-A Phantom Study. J. Appl. Clin. Med. Phys. 2016, 17, 511-531. [CrossRef] [PubMed] 
31. Li, G.; Liu, X.; Dodge, C.T.; Jensen, C.T.; Rong, X.J. A Noise Power Spectrum Study of a New Model-Based Iterative Reconstruction System: Veo 3.0. J. Appl. Clin. Med. Phys. 2016, 17, 428-439. [CrossRef]

32. Andersen, H.K.; Völgyes, D.; Martinsen, A.C.T. Image Quality with Iterative Reconstruction Techniques in CT of the Lungs-A Phantom Study. Eur. J. Radiol. Open 2018, 5, 35-40. [CrossRef]

33. Barca, P.; Giannelli, M.; Fantacci, M.E.; Caramella, D. Computed Tomography Imaging with the Adaptive Statistical Iterative Reconstruction (ASIR) Algorithm: Dependence of Image Quality on the Blending Level of Reconstruction. Australas. Phys. Eng. Sci. Med. 2018, 41, 463-473. [CrossRef]

34. Willemink, M.J.; Noël, P.B. The Evolution of Image Reconstruction for CT-From Filtered Back Projection to Artificial Intelligence. Eur. Radiol. 2019, 29, 2185-2195. [CrossRef]

35. Pan, T.; Hasegawa, A.; Luo, D.; Wu, C.C.; Vikram, R. Technical Note: Impact on Central Frequency and Noise Magnitude Ratios by Advanced CT Image Reconstruction Techniques. Med. Phys. 2020, 47, 480-487. [CrossRef] [PubMed]

36. Hsieh, J. Adaptive Statistical Iterative Reconstruction. White Papper GE Healthcare; 2008; pp. 1-4.

37. Fan, J.; Yue, M.; Melnyk, R. Benefits of ASiR-V Reconstruction for Reducing Patient Radiation Dose and Preserving Diagnostic Quality in CT Exams. White Papper GE Healthcare; 2014; pp. 1-8.

38. Thibault, J.-B. Veo CT Model-Based Iterative Reconstruction. White Papper GE Healthcare; 2010; pp. 1-12.

39. Richard, S.; Husarik, D.B.; Yadava, G.; Murphy, S.N.; Samei, E. Towards Task-Based Assessment of CT Performance: System and Object MTF across Different Reconstruction Algorithms. Med. Phys. 2012, 39, 4115-4122. [CrossRef]

40. McCollough, C.H.; Yu, L.; Kofler, J.M.; Leng, S.; Zhang, Y.; Li, Z.; Carter, R.E. Degradation of CT Low-Contrast Spatial Resolution Due to the Use of Iterative Reconstruction and Reduced Dose Levels. Radiology 2015, 276, 499-506. [CrossRef]

41. Hsieh, J. Computed Tomography: Principles, Design, Artifacts, and Recent Advances; SPIE PRESS: Bellingham, WA, USA, 2015; ISBN 978-1-62841-825-5.

42. Thibault, J.-B.; Sauer, K.D.; Bouman, C.A.; Hsieh, J. A Three-Dimensional Statistical Approach to Improved Image Quality for Multislice Helical CT. Med. Phys. 2007, 34, 4526-4544. [CrossRef]

43. Hussain, F.A.; Mail, N.; Shamy, A.M.; Alghamdi, S.; Saoudi, A. A Qualitative and Quantitative Analysis of Radiation Dose and Image Quality of Computed Tomography Images Using Adaptive Statistical Iterative Reconstruction. J. Appl. Clin. Med. Phys. 2016, 17, 419-432. [CrossRef]

44. Kim, H.G.; Chung, Y.E.; Lee, Y.H.; Choi, J.-Y.; Park, M.-S.; Kim, M.-J.; Kim, K.W. Quantitative Analysis of the Effect of Iterative Reconstruction Using a Phantom: Determining the Appropriate Blending Percentage. Yonsei Med. J. 2015, 56, 253. [CrossRef]

45. Kwon, H.; Cho, J.; Oh, J.; Kim, D.; Cho, J.; Kim, S.; Lee, S.; Lee, J. The Adaptive Statistical Iterative Reconstruction-V Technique for Radiation Dose Reduction in Abdominal CT: Comparison with the Adaptive Statistical Iterative Reconstruction Technique. Br. J. Radiol. 2015, 88, 20150463. [CrossRef]

46. Benz, D.C.; Gräni, C.; Mikulicic, F.; Vontobel, J.; Fuchs, T.A.; Possner, M.; Clerc, O.F.; Stehli, J.; Gaemperli, O.; Pazhenkottil, A.P.; et al. Adaptive Statistical Iterative Reconstruction-V: Impact on Image Quality in Ultralow-Dose Coronary Computed Tomography Angiography. J. Comput. Assist. Tomogr. 2016, 40, 958-963. [CrossRef]

47. So, A.; Imai, Y.; Nett, B.; Jackson, J.; Nett, L.; Hsieh, J.; Wisenberg, G.; Teefy, P.; Yadegari, A.; Islam, A.; et al. Technical Note: Evaluation of a 160-Mm/256-Row CT Scanner for Whole-Heart Quantitative Myocardial Perfusion Imaging. Med. Phys. 2016, 43, 4821-4832. [CrossRef]

48. Racine, D.; Ott, J.G.; Ba, A.; Ryckx, N.; Bochud, F.O.; Verdun, F.R. Objective Task-Based Assessment of Low-Contrast Detectability in Iterative Reconstruction. Radiat. Prot. Dosim. 2016, 169, 73-77. [CrossRef]

49. Kim, H.G.; Lee, H.-J.; Lee, S.-K.; Kim, H.J.; Kim, M.-J. Head CT: Image Quality Improvement with ASIR-V Using a Reduced Radiation Dose Protocol for Children. Eur. Radiol. 2017, 27, 3609-3617. [CrossRef]

50. Gatti, M.; Marchisio, F.; Fronda, M.; Rampado, O.; Faletti, R.; Bergamasco, L.; Ropolo, R.; Fonio, P. Adaptive Statistical Iterative Reconstruction-V versus Adaptive Statistical Iterative Reconstruction: Impact on Dose Reduction and Image Quality in Body Computed Tomography. J. Comput. Assist. Tomogr. 2018, 42, 191-196. [CrossRef] [PubMed]

51. Goodenberger, M.H.; Wagner-Bartak, N.A.; Gupta, S.; Liu, X.; Yap, R.Q.; Sun, J.; Tamm, E.P.; Jensen, C.T. Computed Tomography Image Quality Evaluation of a New Iterative Reconstruction Algorithm in the Abdomen (Adaptive Statistical Iterative Reconstruction-V) a Comparison with Model-Based Iterative Reconstruction, Adaptive Statistical Iterative Reconstruction, and Filtered Back Projection Reconstructions. J. Comput. Assist. Tomogr. 2018, 42, 184-190. [CrossRef]

52. Lim, K.; Kwon, H.; Cho, J.; Oh, J.; Yoon, S.; Kang, M.; Ha, D.; Lee, J.; Kang, E. Initial Phantom Study Comparing Image Quality in Computed Tomography Using Adaptive Statistical Iterative Reconstruction and New Adaptive Statistical Iterative Reconstruction V. J. Comput. Assist. Tomogr. 2015, 39, 443-448. [CrossRef]

53. Tang, H.; Yu, N.; Jia, Y.; Yu, Y.; Duan, H.; Han, D.; Ma, G.; Ren, C.; He, T. Assessment of Noise Reduction Potential and Image Quality Improvement of a New Generation Adaptive Statistical Iterative Reconstruction (ASIR-V) in Chest CT. Br. J. Radiol. 2018, 91, 20170521. [CrossRef]

54. Euler, A.; Solomon, J.; Marin, D.; Nelson, R.C.; Samei, E. A Third-Generation Adaptive Statistical Iterative Reconstruction Technique: Phantom Study of Image Noise, Spatial Resolution, Lesion Detectability, and Dose Reduction Potential. Am. J. Roentgenol. 2018, 210, 1301-1308. [CrossRef]

55. De Marco, P.; Origgi, D. New Adaptive Statistical Iterative Reconstruction ASiR-V: Assessment of Noise Performance in Comparison to ASiR. J. Appl. Clin. Med. Phys. 2018, 19, 275-286. [CrossRef] 
56. Viry, A.; Aberle, C.; Racine, D.; Knebel, J.-F.; Schindera, S.T.; Schmidt, S.; Becce, F.; Verdun, F.R. Effects of Various Generations of Iterative CT Reconstruction Algorithms on Low-Contrast Detectability as a Function of the Effective Abdominal Diameter: A Quantitative Task-Based Phantom Study. Phys. Med. 2018, 48, 111-118. [CrossRef]

57. Chen, L.; Jin, C.; Li, J.; Wang, G.; Jia, Y.; Duan, H.; Pan, N.; Guo, J. Image Quality Comparison of Two Adaptive Statistical Iterative Reconstruction (ASiR, ASiR-V) Algorithms and Filtered Back Projection in Routine Liver CT. Br. J. Radiol. 2018, 91, 20170655 [CrossRef]

58. Tang, H.; Liu, Z.; Hu, Z.; He, T.; Li, D.; Yu, N.; Jia, Y.; Shi, H. Clinical Value of a New Generation Adaptive Statistical Iterative Reconstruction (ASIR-V) in the Diagnosis of Pulmonary Nodule in Low-Dose Chest CT. Br. J. Radiol. 2019, 92, 20180909. [CrossRef]

59. Han, W.K.; Na, J.C.; Park, S.Y. Low-Dose CT Angiography Using ASiR-V for Potential Living Renal Donors: A Prospective Analysis of Image Quality and Diagnostic Accuracy. Eur. Radiol. 2020, 30, 798-805. [CrossRef]

60. International Atomic Energy Agency. Quality Assurance Programme for Computed Tomography: Diagnostic and Therapy Applications; IAEA: Vienna, Austria, 2012; ISBN 978-92-0-128910-0.

61. European Commission. Criteria for Acceptability of Medical Radiological Equipment Used in Diagnostic Radiology, Nuclear Medicine and Radiotherapy; Radiation Protection; Publications Office of the European Union: Luxembourg, 2013; ISBN 978-92-79-27747-4.

62. Dance, D.R. (Ed.) Diagnostic Radiology Physics: A Handbook for Teachers and Students; STI/PUB; International Atomic Energy Agency: Vienna, Austria, 2014; ISBN 978-92-0-131010-1.

63. Riederer, S.J.; Pelc, N.J.; Chesler, D.A. The Noise Power Spectrum in Computed X-Ray Tomography. Phys. Med. Biol. 1978, 23, 446-454. [CrossRef]

64. Friedman, S.N.; Fung, G.S.K.; Siewerdsen, J.H.; Tsui, B.M.W. A Simple Approach to Measure Computed Tomography (CT) Modulation Transfer Function (MTF) and Noise-Power Spectrum (NPS) Using the American College of Radiology (ACR) Accreditation Phantom. Med. Phys. 2013, 40, 051907. [CrossRef]

65. Samei, E.; Bakalyar, D.; Boedeker, K.; Brady, S.; Fan, J.; Leng, S.; Myers, K.; Popescu, L.; Ramirez-Giraldo, J.C.; Ranallo, F.; et al. Performance Evaluation of Computed Tomography Systems-The Report of AAPM Task Group 233; AAPM: Alexandria, VA, USA, 2019.

66. The Phantom Laboratory. Catphan ${ }^{\circledR} 504$ Manual; The Phantom Laboratory: Salem, NY, USA, 2013.

67. Li, K.; Tang, J.; Chen, G.-H. Statistical Model Based Iterative Reconstruction (MBIR) in Clinical CT Systems: Experimental Assessment of Noise Performance. Med. Phys. 2014, 41, 041906. [CrossRef]

68. Solomon, J.; Samei, E. Quantum Noise Properties of CT Images with Anatomical Textured Backgrounds across Reconstruction Algorithms: FBP and SAFIRE. Med. Phys. 2014, 41, 091908. [CrossRef]

69. Solomon, J.; Wilson, J.; Samei, E. Characteristic Image Quality of a Third Generation Dual-Source MDCT Scanner: Noise, Resolution, and Detectability. Med. Phys. 2015, 42, 4941-4953. [CrossRef] [PubMed]

70. Funama, Y.; Taguchi, K.; Utsunomiya, D.; Oda, S.; Katahira, K.; Tokuyasu, S.; Yamashita, Y. Image Quality Assessment of an Iterative Reconstruction Algorithm Applied to Abdominal CT Imaging. Phys. Med. 2014, 30, 527-534. [CrossRef]

71. Geyer, L.L.; Körner, M.; Harrieder, A.; Mueck, F.G.; Deak, Z.; Wirth, S.; Linsenmaier, U. Dose Reduction in 64-Row Whole-Body CT in Multiple Trauma: An Optimized CT Protocol with Iterative Image Reconstruction on a Gemstone-Based Scintillator. Br. J. Radiol. 2016, 89, 20160003. [CrossRef]

72. Brady, S.L.; Yee, B.S.; Kaufman, R.A. Characterization of Adaptive Statistical Iterative Reconstruction Algorithm for Dose Reduction in CT: A Pediatric Oncology Perspective. Med. Phys. 2012, 39, 5520-5531. [CrossRef] 